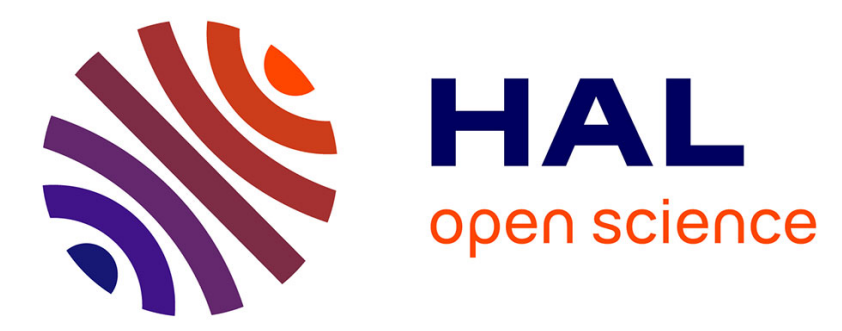

\title{
Les syndicats de la Fonction publique et les réformes managériales depuis 2002
}

\author{
Jeanne Siwek-Pouydesseau
}

\section{To cite this version:}

Jeanne Siwek-Pouydesseau. Les syndicats de la Fonction publique et les réformes managériales depuis 2002. Revue française d'administration publique, 2009, 4 (132), pp.745-756. halshs-00480112

\section{HAL Id: halshs-00480112 \\ https://shs.hal.science/halshs-00480112}

Submitted on 3 May 2010

HAL is a multi-disciplinary open access archive for the deposit and dissemination of scientific research documents, whether they are published or not. The documents may come from teaching and research institutions in France or abroad, or from public or private research centers.
L'archive ouverte pluridisciplinaire HAL, est destinée au dépôt et à la diffusion de documents scientifiques de niveau recherche, publiés ou non, émanant des établissements d'enseignement et de recherche français ou étrangers, des laboratoires publics ou privés. 


\title{
Les syndicats de la Fonction publique et les réformes managériales depuis
}

\author{
Jeanne Siwek-Pouydesseau \\ Directrice de Recherche honoraire au CNRS. \\ Centre d'Etudes et de Recherches de Sciences Administratives et Politiques \\ (CERSA. CNRS-Paris2).
}

Depuis des décennies, tels des Cassandre, des syndicats annoncent la catastrophe où mèneraient les déréglementations libérales ou néolibérales. D'autres ont préféré accompagner des réformes jugées indispensables, en tentant d'en limiter les effets les plus pervers pour les agents ${ }^{1}$. Beaucoup d'organisations ont surtout critiqué une idéologie longtemps cachée, mais qui est devenue de plus en plus explicite au cours du temps, renforcée par des expériences étrangères souvent sélectionnées sans en attendre les résultats définitifs. L'irrésistible ascension du Budget dans la gestion de la fonction publique en est l'aboutissement logique.

Luc Rouban a relevé qu'en matière d'emploi public les statistiques de l'OCDE sont loin d'être fiables ${ }^{2}$. Une nouvelle méthodologie de collecte de données comparables a donc été entreprise, mais une lecture attentive du précieux document de travail de $1^{\prime} \mathrm{OCDE}^{3}$ sur la question montre qu'on est toujours dans l'incapacité de faire des comparaisons pertinentes. Jusqu'ici, ces comparaisons, dans un sens ou un autre, ont été surtout d'ordre idéologique.

Récemment $^{4}$, le Premier Président de la Cour des Comptes remarquait que le non renouvellement d'un fonctionnaire sur deux partant à la retraite aboutirait, déductions comprises, à environ deux milliards d'économies par an, face aux 70 milliards de manque à gagner venant des différentes niches et dépenses fiscales. La réduction des dépenses de fonctionnement n'en était, pour lui, pas moins nécessaire. Lorsque les syndicats font le même

\footnotetext{
${ }^{1}$ Siwek-Pouydesseau (Jeanne). Les syndicats des fonctions publiques au XXe siècle, Paris, Berger-Levrault, 2001, 312 p.

${ }^{2}$ Rouban (Luc). La fonction publique (3ème édition), Paris, La Découverte, 2009, p. 36.

${ }^{3}$ Pilichowski (Elsa) et Turkisch (Edouard), «L'emploi dans le secteur des administrations publiques sous l'angle des coûts de production des biens et des services dans le domaine public », Documents de travail sur la gouvernance publique, $\mathrm{n}^{\circ}$ 8, Editions OCDE, 2008, $66 \mathrm{p}$.

${ }^{4}$ Rencontres de la Modernisation de l'Etat, 8 juillet 2009, Acteurs Publics. www.acteurspublics.com

Voir : Cour des Comptes, Rapport sur la situation et les perspectives des Finances publiques, juin 2009, 133 p.
} 
calcul pour relativiser ce remède miracle, prôné depuis la IIIe République par les officines proches du patronat, le raisonnement ne devrait pas en être disqualifié pour autant.

Mais que certaines critiques syndicales soient souvent systématiques, quelles que soient les réformes proposées, est un fait, renforçant les luttes idéologiques des deux côtés. Les organisations syndicales font, malgré tout, un sérieux travail pour disséquer l'avalanche des projets de réformes, qu'ils aboutissent ou pas. Il leur faut sans cesse sur le métier remettre leur ouvrage, expliquer le contenu des textes, supputer leurs conséquences concrètes pour les agents et argumenter les raisons pour lesquelles ils s'y opposent ou pas. Il faudrait ensuite qu'ils fassent des propositions alternatives pour être crédibles mais il est vrai que souvent ces propositions s'écartent peu, soit d'une amélioration à la marge du système antérieur, soit des idées les plus couramment admises dans l'encadrement ${ }^{5}$. Le plus intéressant est de détecter les évolutions syndicales lorsqu'elles ont existé mais, aujourd'hui, les nouvelles règles concernant la représentativité risquent de bouleverser l'échiquier syndical.

Nous ferons un panorama des argumentaires de la presse syndicale des trois principales organisations confédérées dans la fonction publique de l'Etat depuis 2002, hormis les enseignants qui ont des problèmes particuliers ${ }^{6}$. Les autres organisations reprennent peu ou prou les mêmes démonstrations à partir de ces trois exemples, en mettant l'accent sur certains points en fonction des préoccupations de leurs mandants. Ces argumentaires ne sont pas toujours lus par les militants, et encore moins par les adhérents, cependant leur répétition finit par imprégner la culture syndicale et sert à définir les identités des différentes organisations, en compétition lors des élections mais qui, sur l'essentiel, ont beaucoup d'intérêts communs ${ }^{7}$. Ces textes ne sont pas, en priorité, destinés à convaincre les pouvoirs publics mais tentent de maintenir une pression pour mobiliser les troupes le moment venu. Ils sont le plus souvent d'une grande cohérence logique, toutefois les non-dits sont tout aussi significatifs. Nous n'aborderons pas les revendications salariales ou concernant les retraites, longtemps cœur de cible traditionnel des syndicats, qui suscitent la plupart des mouvements de grèves et sont de plus en plus difficiles à défendre dans un contexte de crise. La défense de l'emploi est, en effet, devenue prioritaire et les réductions de plus en plus drastiques des effectifs laissent peu de marge à un dialogue serein.

\footnotetext{
${ }^{5}$ A noter que la CFDT reste la plus représentative des trois confédérations en catégorie A.

${ }^{6}$ Résultats des élections professionnelles dans la Fonction publique de l'Etat au 31 décembre 2008 : FSU 20,2\% ; CGT 15,6\% ; UNSA 16,8\% ; FO 13\% ; CFDT 11,3\%. Voir :

Rapport annuel sur l'état de la Fonction publique, vol. 1. Faits et chiffres 2008-2009, DGAFP. p. 522.

${ }^{7}$ Comme le montrent de fréquents communiqués de presse communs à tout ou partie des organisations.
} 


\section{La Fédération générale des Fonctionnaires FO, pour une résistance sans concession ${ }^{8}$}

Après la déclaration de politique générale du gouvernement Raffarin, la Nouvelle Tribune de juillet 2002 titrait «Vers l'Etat minimum ? ». Pour la Fédération générale des Fonctionnaires FO, ce programme libéral rappelait celui du député Gérard Longuet en 1980. Alors que la fonction publique devrait illustrer la conception républicaine de l'égalité des citoyens et qu'elle ne pourrait être remise en cause au nom des bienfaits supposés de la concurrence et de la compétitivité. Cette réforme libérale de l'Etat serait un choix politique et partisan, faisant fi de l'intérêt général ainsi que de l'indépendance et de la neutralité garanties par le statut des fonctionnaires. L'égalité d'accès aux services publics, la péréquation des charges et la redistribution par l'impôt seraient remis en cause. De même, la décentralisation et la régionalisation iraient à l'encontre de la tradition centralisatrice assurant l'unité et l'indivisibilité de la République. D'où l'opposition de Force Ouvrière à une Europe des régions, ainsi qu'aux transferts de compétences et à une fiscalité régionale. En réaction aux vœux du président Chirac, la Fédération générale des Fonctionnaires affirmait à nouveau qu'il ne s'agissait pas, en réalité, de rendre le service public plus efficace mais de poursuivre une orientation politique d'inspiration libérale, qui tendrait à désengager l'Etat de ses missions et à privatiser le service public. Pour limiter le budget de l'Etat, il faudra donc diminuer les effectifs, le niveau des rémunérations, le coût des retraites, etc. Aussi, lors de son congrès de 2003, la FGF entendait «reconquérir les valeurs républicaines ». La décentralisationrégionalisation risquait d'être une étape vers la privatisation, car les contraintes européennes pour restreindre les dépenses budgétaires finiront par s'appliquer aux collectivités territoriales.

Dans cette logique libérale, la loi organique relative aux lois de Finances (LOLF) amènera les services à définir les priorités de leurs missions en fonction des moyens dont ils disposeront, alors que, pour Force Ouvrière, les fonctionnaires n'ont aucune légitimité à en choisir eux-mêmes les priorités. Le libéralisme économique va substituer des automatismes budgétaires à la décision politique et donc à la démocratie. On passera ainsi d'une administration de service public à des centres de profits, appuyés sur la contractualisation comme mode de gestion.

Les critiques étaient évidemment encore plus virulentes concernant les évolutions de la fonction publique et du statut. En effet, toutes les dérives étaient possibles pour contourner les

\footnotetext{
${ }^{8}$ Voir La Nouvelle Tribune, Fédération générale des Fonctionnaires FO, trimestriel, 2002-2009.
} 
garanties statutaires. Pour Force Ouvrière, il n'était pas question d'accepter un système d'évaluation lié à la performance, ni des formules d'intéressement faisant que le point d'indice ne représenterait qu'une partie de plus en plus réduite du traitement global. Les projets de disparition des corps au profit de cadres d'emplois étaient également récusés et rendraient les commissions administratives paritaires obsolètes. Plus précisément, des enveloppes globales déléguées à des gestionnaires locaux mettront en péril le caractère égalitaire du service public. En fusionnant un plafond unique d'emplois d'agents titulaires et non titulaires, on accentuera la précarité et, en fondant dans une même masse salariale les traitements principaux et les primes, on ira vers une individualisation des rémunérations. Les ratios entre agents promus et promouvables videront les commissions administratives paritaires de leur rôle en matière d'avancement. Enfin, la transformation d'une fonction publique de carrière en fonction publique de métiers ne permettra pas au nouveau système de s'améliorer après la phase de rodage.

Les nouvelles conceptions du dialogue social étaient aussi réfutées. En effet, FO ne revendique pas de cogérer l'Etat et considère que les organisations syndicales, tout comme les fonctionnaires, n'ont pas de légitimité pour cela. Il n'était donc pas question de transformer les comités techniques paritaires en comités d'entreprises élus, ni en comités tripartites avec des usagers, car la représentation d'intérêts particuliers s'opposerait à l'intérêt général. D'autant que les pouvoirs publics ont toujours tendance à faire cautionner par les syndicats ce qui est déjà décidé. Par ailleurs, la FGF refusait de valider des accords qui, d'une manière ou d'une autre, entérineraient des restrictions budgétaires. Force Ouvrière n'a donc pas signé le relevé des conclusions sur le dialogue social en juin 2008 car elle s'oppose, notamment, aux accords majoritaires calqués sur ceux du secteur privé et à la généralisation de l'élection aux comités techniques, qui devraient rester paritaires.

Le rapport moral au congrès de 2006 ajoutait à ces critiques régulièrement reprises, le «vaste chantier de déconstruction » entrepris par la loi de décentralisation et par les audits de modernisation du ministre délégué au Budget et à la réforme de l'Etat, avec une direction générale composée de seulement 40\% d'agents publics sur 160 personnes, dépossédant la direction générale de l'Administration et de la Fonction publique de ses missions en matière de réforme de l'Etat. La résolution générale du congrès condamnait la logique du traité de Maastricht, refusait le dogme du non remplacement des fonctionnaires partant à la retraite, rejetait toute externalisation, privatisation ou sous-traitance sous-tendues par la volonté de réduire les effectifs, et s'opposait au recrutement de personnels non titulaires. Le congrès réaffirmait l'exigence d'un service public de l'Etat «puissant et impartial, non tributaire de 
données mercantiles et des conjonctures politiciennes, garantie de l'égalité de traitement des citoyens, de la cohésion du tissu social et support des idéaux démocratiques républicains ». Il dénonçait les tentatives de transposition du modèle anglo-saxon en France, ainsi que la campagne permanente opposant le secteur privé au secteur public. Le congrès condamnait à nouveau la LOLF et demandait l'abandon des notions d'objectifs et d'indicateurs, qui conduiraient d'une culture de moyens à une culture de résultats, ainsi que l'abrogation de la fongibilité asymétrique des crédits incitant à l'externalisation et à la privatisation. Il refusait enfin l'introduction de critères de productivité et de rentabilité dans la fonction publique, de même que la séparation entre les missions d'autorité et de puissance publique et celles de production et d'exécution du service public.

La Révision générale des Politiques publiques (RGP) fut encore plus mal accueillie, «élaborée en catimini » par quelques hauts fonctionnaires et conseillers privés, et dont le résultat sera «l'inégalité généralisée selon les ressources du client ». Pour la FGF-FO, il faut donc résister, car la RGPP n'est, ni une simple technique ni une mode du moment, mais une révolution totale des grands schémas directeurs du Service public. En effet, si la LOLF faisait craindre des économies au détriment des budgets ministériels de fonctionnement, la RGPP va beaucoup plus loin. Elle bouleverse l'organisation administrative et le contenu de l'action publique, aboutissant à une configuration libérale de l'administration territoriale et à un dessaisissement de l'Etat.

Par ailleurs, dans le cadre de la RGPP, le projet de loi sur la mobilité des fonctionnaires votée en 2008 au Sénat allait démanteler la fonction publique. Avec la suppression de 30.000 emplois en 2009 et le non remplacement d'un fonctionnaire sur deux, c'était un vaste plan social qui s'annonçait, complété par des mobilités géographiques forcées et des incitations au départ. FO dénonçait les transferts de missions ou de compétences ainsi que la fermeture des services de proximité, sans qu'aucun débat public n'ait été organisé. Cette loi disposait que le fonctionnaire dont l'emploi serait susceptible d'être supprimé serait placé en réorientation professionnelle et, s'il refusait trois propositions de postes, il pourrait être placé en disponibilité d'office ou admis à la retraite ${ }^{9}$. Etaient également prévus des emplois permanents à temps non complet cumulés, le recrutement d'agents non titulaires, et d'intérimaires pour assurer le remplacement momentané de fonctionnaires, qui faisaient craindre des contrats de droit privé ou le recours à des agences d'intérim.

\footnotetext{
${ }^{9}$ L'article 7 de la loi définitivement votée précise : trois offres d'emploi public «correspondant à son grade et à son projet personnalisé d'évolution professionnelle, et en tenant compte de sa situation de famille et de son lieu de résidence habituel ».
} 
Aussi, la FGF-FO avait-elle lancé, en mars 2009, une pétition contre la RGPP et la loi sur la mobilité des fonctionnaires. Elle jugeait, en effet, inopportun de diminuer de façon drastique les effectifs de la fonction publique de l'Etat pendant que se développait une grave crise financière, économique et sociale, et alors que la France semblait mieux résister que d'autres grâce à des services publics forts. Aucun débat public n'avait permis d'exposer les tenants et aboutissants de cette loi, dont il faudrait, au minimum, retirer les articles 7 et 10. La pétition demandait également la fin des suppressions de postes dans la fonction publique de l'Etat. La loi relative à la mobilité et aux parcours professionnels dans la fonction publique a été promulguée le 3 août 2009 (JO du 6 août 2009).

\section{L'Union générale des fédérations de Fonctionnaires CGT, pour une fonction publique citoyenne $^{10}$}

A l'occasion de son $23^{\text {ème }}$ congrès de 2003, l'UGFF avait préparé un long document définissant sa conception de l'Etat. Elle se référait à la Déclaration des Droits de l'Homme de 1789 établissant la prééminence de la loi, l'égalité d'accès aux emplois publics, la responsabilité des agents publics face à la société, le paiement de l'impôt, ainsi que sa transparence et sa progressivité. Mais la conception du Service public qui s'était développée au XIXe et au XXe siècle avait subi une offensive libérale dans le monde et en Europe. Ainsi, le rapport Picq de 1994 avait dessiné les contours d'un Etat réduit à des missions de régulation, dont le nouveau rôle serait de structurer le fonctionnement de la société sur la logique du marché. D’où la réduction des impôts et de l'emploi public, ainsi que des acquis sociaux. D'où aussi les transferts de compétences aux collectivités locales sans transferts de moyens, les contraintes budgétaires étant liées aux revendications du MEDEF.

Pour l'UGFF, avec la $\boldsymbol{L} \boldsymbol{O L F}$, le risque était grand de voir se développer une gestion managériale des services en direction d'usagers transformés en clients. Pour cela, les garanties statutaires des agents étaient, pour le gouvernement, un obstacle à lever. La LOLF répondait à une logique de résultat, les indicateurs de gestion mis en place découlaient d'une politique néolibérale de réduction des coûts, qui sous-tendait l'ensemble des réformes de l'Etat. Ainsi, les dépenses de personnels constituaient toujours la véritable variable d'ajustement. Cette loi a été adoptée sans réel débat public ni expertise, alors que les missions publiques sont un bien public qui devrait donner lieu à un débat démocratique, et que le critère d'efficacité sociale

\footnotetext{
${ }^{10}$ Voir Fonction Publique, UGFF-CGT, mensuel, 2002-2009.
} 
devrait être la finalité de la gestion publique. Par ailleurs, le Parlement ne serait pas en mesure de peser sur la présentation du budget.

La CGT revendiquait, au contraire, une fonction publique de la Nation pour l'usagercitoyen, car la satisfaction de ses droits ne pouvait être laissée au libre jeu du marché. La décentralisation devait se situer dans un cadre national réaffirmé, avec la construction d'une Europe différente, plus transparente et démocratique. Il fallait donc agir pour des réformes qui amèneraient des progrès en termes de services publics, de solidarité entre les territoires, et combattre les politiques qui conduisent à des privatisations rampantes. L'UGFF présentait ainsi un véritable programme gouvernemental de réforme de l'Etat, autour d'une « déconcentration démocratique » et d'un fonctionnaire-citoyen. Il faudrait, par ailleurs, définir une politique dynamique de l'emploi, en réponse aux besoins sociaux et aux attentes des personnels, sortir du pacte de stabilité, revenir sur la baisse des prélèvements obligatoires et réformer la fiscalité. L'UGFF se réclamait donc d'un syndicalisme de transformation sociale, avec la construction de rapports de force mettant l'usager au cœur de cette démarche. Aussi les syndicats devraient-ils s'organiser face aux stratégies ministérielles de réforme, qui font le lien entre Réforme de l'Etat et mise en œuvre de la LOLF. L'UGFF s'interrogeait sur ce qui restera de la grille indiciaire et des commissions administratives paritaires qui n'auront plus à examiner les décisions individuelles. Or, seules des garanties unifiées permettent la mobilisation au sein de la fonction publique entre services, qu'ils relèvent de l'Etat ou des collectivités territoriales. La CGT proposait donc la création de commissions des services publics à tous les niveaux : quartiers, pays, agglomérations, département, région... où les représentants des usagers devront prendre toute leur place. Finalement, elle demandait un statut rénové et unifié, avec des passerelles fortes entre ses trois versants.

Cependant, l'UGFF ne refusait pas toute discussion sur l'évolution de la gestion publique et participa aux travaux de l'Observatoire de l'emploi public, contrairement à Force Ouvrière. Comme les autres participants, elle reconnut la qualité des travaux fournis. Si les orientations de la gestion prévisionnelle des effectifs, des emplois et des compétences (GEPEEC) étaient discutables, les outils d'information avaient été mis à la disposition de tous en temps utile. Certes l'UGFF disait ne pas être dupe car on s'orientait vers un répertoire des métiers, mais les critiques syndicales avaient permis d'en limiter les dérives. En conclusion, elle n'était pas, par principe, hostile au répertoire qui assurait une meilleure lisibilité des emplois mais, si les garanties collectives devaient être rabaissées, elle s'y opposerait.

Pour l'UGFF, le statut général des fonctionnaires restait un gage de qualité et un outil de gestion moderne à condition de rendre la fonction publique attractive en améliorant le pouvoir 
d'achat et la grille unique des rémunérations, sans oublier les retraites et l'action sociale. Sa remise en cause n'était pas négociable, alors que le recours aux non titulaires était élargi, que les corps seraient regroupés par filières pour aboutir à une fonction publique de métiers. La gestion par la performance et la rémunération au mérite conduisaient également à une remise en question du statut. Devaient rester au cœur des garanties, les corps, les grades, les catégories, les concours, les CAP, la grille unique... La loi sur la modernisation de la fonction publique, votée en février 2007 à la suite de l'accord signé en janvier 2006 par la CFDT, la CFTC et l'UNSA, mais rejeté par la CGT, FO et la FSU, concernait notamment la formation professionnelle, les déroulements de carrière et l'évaluation. L'UGFF soulignait que le projet n'avait pas obtenu un soutien majoritaire des syndicats et que cette «modernisation » était, le plus souvent, un recul.

Le discours du Président de la République à l'IRA de Nantes en septembre 2007 sur la fonction publique renouvela les critiques de l'UGFF comme celles des autres organisations. La CGT récusait le principe d'une fonction publique moins nombreuse et mieux payée, le pécule pour ceux qui accepteraient de quitter leur poste de fonctionnaire, la remise en cause de la carrière et du concours, les corps remplacés par des métiers, le choix entre le statut et le contrat pour les nouveaux recrutements, l'individualisation des rémunérations et de l'évaluation, les concours supprimés pour la catégorie C... L'UGFF proposait une fois encore des pistes alternatives, à commencer par taxer davantage le capital, et une « véritable réforme fiscale de progrès ", l'arrêt des suppressions d'emplois, un débat contradictoire sur les missions de la fonction publique, un plan massif de titularisation, une augmentation de la valeur du point d'indice, l'amélioration de la grille indiciaire, la ré-indexation des pensions sur les salaires...

La CGT accepta de participer aux quatre conférences organisées sur la fonction publique, au moins pour information et pour pouvoir rendre compte à ses adhérents des projets en cours, mais aussi de manière à «brider les velléités les plus libérales » et à gagner le plus grand nombre à une «conception progressiste» de la fonction publique. Contrairement à Force Ouvrière, la CGT pratiquait de moins en moins la politique de la chaise vide.

A l'occasion du Livre blanc remis en 2008 par J. L. Silicani, l'UGFF rappelait ses objections et demandait un bilan contradictoire des déréglementations déjà opérées. Elle signalait qu'au Canada, donné en exemple, il y avait plus d'emplois qu'avant les coupes budgétaires et que, de 1980 à 2006, les dépenses n'avaient baissé que de 2\% du PIB. De même, une étude du Centre d'analyse stratégique sur l'évolution de l'emploi public dans les pays développés constatait qu'après une compression, le volume des emplois avait été reconstitué. L'Union des 
ingénieurs et cadres CGT de la fonction publique organisait alors un large débat public au cours duquel un directeur de cabinet ministériel affirmait que le statut de 1946 «sentait le pétainisme ravaudé, avec ses statuts et ses corps $»^{11}$, le bon exemple venant de l'Italie où la fonction publique a été alignée sur le droit du travail. Pour lui, il ne faudrait d'ailleurs pas parler d'indépendance du fonctionnaire puisque celui-ci est là pour servir. Tout au contraire, face à la «contre-réforme» d'une fonction publique organisée en métiers, face à la suppression du classement de l'ENA faisant craindre un nouveau mode de cooptation, l'UGFF estimait que les cadres devaient pouvoir disposer de réelles marges de manœuvre et exprimer leur opinion dans la façon de mettre en œuvre l'intérêt général.

Concernant le dialogue social, la CGT se prononçait pour de nouveaux droits syndicaux et pour l'organisation de la concertation : «Si nous ne sommes pas partisans de la cogestion (voire de la codécision) incompatible avec la conception que nous avons de la fonction publique, de son lien avec la Nation et de la responsabilité de ses représentants, nous entendons que l'opinion des personnels, leurs revendications, puissent s'exprimer, peser réellement, être pris en compte $»^{12}$. Alors que la concertation existante n'était qu'une formalité, le pouvoir d'initiative syndicale en matière de négociation devait être élargi. De même, des prérogatives nouvelles devaient être données aux comités techniques sur l'ensemble des questions liées à la LOLF, notamment sur l'emploi public, l'évaluation des indicateurs mesurant l'efficacité sociale et la qualité du service. Un accord ne pouvait être valide que s’il était approuvé par la majorité des personnels. La CGT demandait, en outre, que lorsqu'une majorité refuse un avis favorable, son effet soit suspensif - ce qui a été obtenu. Mais, contrairement à la CFDT, elle considérait qu'il n'était pas opportun de donner une valeur juridique directe à l'accord et qu'il suffisait au gouvernement de s'engager à appliquer ce qu'il avait signé. L'UGFF a ratifié le protocole de Bercy sur le dialogue social en juin 2008, en liaison avec les positions confédérales sur la représentativité. Elle a approuvé la représentation des titulaires et non titulaires dans les comités techniques ainsi que les nouvelles modalités électorales et la création d'un Conseil supérieur commun aux trois fonctions publiques. De même, le champ des négociations sera élargi et la validation des accords par des organisations représentant la majorité des suffrages entériné. En revanche, l'UGFF estimait inacceptable que les comités de suivi d'un accord soient limités aux seuls signataires et que les négociations salariales deviennent triennales. L'évolution du paritarisme, qui remettrait en cause la collégialité des instances comme les CAP, posait

\footnotetext{
${ }^{11}$ Cité dans Fonction Publique, UGFF-CGT, janvier 2009, p. 14.

${ }^{12}$ Fonction Publique, UGFF-CGT, août 2003, p. 42.
} 
également problème. Finalement, le dialogue social a été jugé caricatural dans la mise en œuvre de la RGPP et la CGT a voté contre le projet de loi présenté aux conseils supérieurs en 2009.

Avec la Révision des politiques publiques (RGPP), le nouveau quinquennat avait son emblème, qui finit par regrouper toutes les réformes en cours. Le modèle de la réussite était trouvé au Canada qui, en quatre ans, aurait diminué les crédits des ministères de 5 à $40 \%$ et les effectifs de $16 \%$. Des audits de cabinets privés et d'inspections générales avaient travaillé en secret sur ce qui serait transféré aux collectivités locales ou externalisé, toutes les missions rentables risquant de passer au privé. Une fois encore, était dénoncée l'absence de toute concertation avec les organisations professionnelles. De même, les agences de service public ont été présentées pour expérimentation à la Commission de Modernisation des Services publics de mars 2008, sans consultation préalable, ni rapport clair sur les objectifs. Alors qu'une étude de l' IGPDE $^{13}$ sur les agences, notamment en Grande Bretagne, montrait qu'elles ont peu à voir avec les attentes des usagers. Face à la fusion entre les directions des Impôts et de la Comptabilité publique, l'UGFF proposait la création de maisons des Finances, des services de proximité, en respectant la séparation entre ordonnateurs et comptables, entre assiette et recouvrement. Cependant, la fédération des Finances CGT et ses syndicats étaient prêts à participer aux groupes de travail ministériels. Avec l'apparition de la crise et la poursuite de la RGPP sur sa lancée comme si de rien n'était, la CGT chercha un nouvel angle d'attaque. Non seulement les réformes étaient passées en force, sans évaluation préalable et sans consultation, mais aucune inflexion ne semblait programmée, les réductions de crédits et d'effectifs apparaissant de plus en plus décalées, à contre-emploi et contre-conjoncture. Alors que l'on parlait du retour de l'Etat et du rôle des services publics comme amortisseurs de la crise, la fonction publique devrait devenir un atout.

Pour son congrès de 2009, l'UGFF rappelait qu'elle avait toujours eu la volonté d'associer les usagers et les citoyens à ses propositions, et qu'elle poursuivait une campagne commune sur le Service public avec la FSU, les syndicats Solidaires ainsi que des associations. Elle faisait cependant état de divergences avec sa confédération sur la décentralisation et ses conséquences pour les personnels de l'Education nationale et de l'Equipement. De manière plus large, les réformes de l'administration territoriale de l'Etat ne manqueront pas d'entraîner de douloureuses réorganisations des structures syndicales.

\footnotetext{
${ }^{13}$ Institut de la gestion publique et du développement économique du ministère de l'Economie et des Finances.
} 


\section{L'Union des Fédérations des Fonctions publiques et assimilés CFDT, ou le discours de la méthode}

Lors d'un colloque en $2002^{14}$, le secrétaire général de la CFDT-Cadres regroupant l'encadrement public et privé, François Fayol, administrateur civil au ministère des Finances, précisait sa philosophie de la modernisation administrative, qui devait être une responsabilité partagée. Pour lui, la modernisation des administrations publiques était nécessaire et indispensable à la réforme de l'Etat. Elle devait se construire jour après jour par des agents et des cadres prenant leurs responsabilités, par des organisations syndicales et des responsables administratifs reconnaissant leur légitimité réciproque, acceptant la confrontation de logiques différentes, négociant une évolution du service public, en mettant au centre de la démarche la qualité du service au citoyen. «Nos objectifs ne sont pas ceux de la définition d'un Etat minimal qui ferait de la baisse des effectifs et de la réduction des déficits publics les seules finalités. Ils ne sont pas non plus ceux d'un repli frileux sur des certitudes du passé et des modes d'organisation des services publics immuables ». Il s'agissait donc de rechercher un « mieux d'Etat» facteur de cohésion sociale, et d'améliorer la qualité des services rendus au moindre coût pour la collectivité. En effet, l'Etat régulateur serait un compromis entre une logique libérale de désengagement et la préservation du grand secteur public hérité de l'aprèsguerre. Pour les administrations de l'Etat, les fonctions opérationnelles devront être confiées aux services déconcentrés, le suivi et l'évaluation des résultats pilotés par les administrations centrales. F. Fayol se prononçait pour une nouvelle vague de décentralisation au profit des régions ainsi que pour une déconcentration accrue, y compris des personnels « rendant plus difficile une gestion corporative par l'administration comme par les organisations syndicales ».

La $\boldsymbol{L O L F}$ devait ainsi orienter la gestion vers les résultats et renforcer la transparence, dans une logique de confiance. Mais la modernisation ne pouvait se faire contre les agents et les cadres qui constituent un relais. La rétribution ne devait pas se limiter à des primes mais aussi à l'amélioration des conditions de travail. Il fallait s'appuyer sur une vraie culture de négociation aboutissant au compromis et à la signature, «sans considérer comme immuable ce qui a pu être qualifié d'acquis dans une période antérieure ». Dans cette philosophie participative basée sur la confiance, il fallait définir les enjeux et contreparties : établir un diagnostic en commun entre responsables administratifs et responsables syndicaux, s'accorder

\footnotetext{
${ }^{14}$ « Moderniser les administrations publiques », Cadres CFDT, mai 2002, p. 55-62.
} 
sur les objectifs, rechercher des solutions, évaluer les expérimentations et définir un accordcadre. Les responsables administratifs devront avoir une réelle délégation pour négocier, à condition de clairement distinguer les responsables politiques des responsables administratifs. Ce texte se présentait, en quelque sorte, comme une feuille de route pour le prochain gouvernement.

Deux ans plus tard, la déception était grande ${ }^{15}$, car la modernisation avançait lentement et le dialogue social, de même que la négociation, n'avaient toujours que très peu de place dans les prises de décision. «Lorsqu'un gouvernement ne s'engage pas, faut-il s'étonner des attitudes syndicales de refus et de méfiance ? ». La mise en place de la LOLF apparaissait comme un exercice d'état-major et de nombreux fonctionnaires, y compris les cadres, l'ignoraient. Alors que le credo politique rejoignait les certitudes libérales sur la limitation des prélèvements obligatoires, il faudrait d'abord une réflexion sur l'équité et la justice sociale. Il faudrait ensuite passer d'une culture de la méfiance et du contrôle a priori à une culture de la confiance et du contrôle a posteriori. Il faudrait enfin une fonction Ressources Humaines plus stratégique et plus proche, dans une démarche plus contractuelle que statutaire, et créer des cadres d'emplois ad hoc, par filières de métiers, à la place de trop nombreux corps. En conclusion, le secrétaire général des Cadres CFDT pointait la nécessité absolue d'une pédagogie de la réforme, de la concertation et de la négociation avec les partenaires sociaux. Avec l'élection présidentielle de $2007^{16}$, l'inquiétude de l'UFFA-CFDT se renforça. Mais elle se disait partante pour le cycle des quatre conférences de discussions prévues par le gouvernement sur le pouvoir d'achat, le dialogue social, les parcours professionnels, ainsi que les valeurs, missions et métiers de la fonction publique.

Concernant le dialogue social, la CFDT reprenait la demande déjà formulée en 2006, à savoir que l'audience des syndicats soit mesurée à partir des élections aux comités techniques paritaires à la fois pour les titulaires et les non titulaires, que les accords soient approuvés par des organisations représentant une majorité d'agents et prennent une valeur juridique s'imposant aux employeurs. Une instance inter-fonctions publiques devrait examiner les projets transversaux aux conseils supérieurs des trois fonctions publiques, le rôle des CTP serait renforcé dans le cadre de la LOLF, les CAP continueraient à débattre des carrières individuelles, mais la gestion des corps reviendrait aux Comités paritaires. Des CAP locales ou communes à plusieurs corps ou familles de métiers pourraient être envisagées. Après avoir

\footnotetext{
${ }^{15}$ Colloque CFDT-Cadres et CFDT-Fonctions publiques. LOLF et MODERFIE, 11 juin 2004. www.cadresplus.net

${ }^{16}$ Fonctions Publiques Informations. CFDT, 31 octobre 2007, p. 3-4.
} 
signé les accords de Bercy en juin 2008, la CFDT a approuvé le projet de loi présenté aux conseils supérieurs.

Enfin, l'UFFA était dubitative concernant le lancement de la Révision générale des Politiques publiques (RGPP) qui conduisait à la réduction des dépenses de l'Etat, «comprendre les réductions d'effectifs » et à plus d'efficacité de l'intervention publique, «c'est surtout une démarche de l'exécutif qui prendra seul ses responsabilités dans les décisions de réformes ! ». Dans ce programme, il y avait peu de place pour le dialogue social, si ce n'est que les secrétaires généraux des ministères devront rencontrer les organisations syndicales pour faire le point sur cette RGPP... De fait, la rapidité des échéances prévues interdisait une réelle concertation. Sur les parcours professionnels ${ }^{17}$, il apparut vite que, en liaison avec la RGPP, le gouvernement était en priorité intéressé par la mobilité et pressé d'inventer une sorte d'intérim dans les fonctions publiques. L'UFFA rappelait encore une fois qu'on ne modernise pas contre les personnels, que le temps du dialogue social peut être long et elle se disait déçue et inquiète.

La nouvelle secrétaire générale de l'UFFA-CFDT en 2008, Brigitte Jumel, reprit ces thèmes en insistant sur une réforme nécessaire, à condition de s'en donner les moyens ${ }^{18}$. Décentralisation, déconcentration et LOLF devaient donner lieu à une large concertation pour définir les objectifs et la stratégie de changement et, pour cela, il fallait refonder les relations sociales, sécuriser les parcours des personnels titulaires et non titulaires en rendant les carrières attractives. Il fallait aussi valoriser la fonction GRH en la déconcentrant, passer d'une culture de la méfiance à une culture de la confiance, et individualiser les parcours sans remettre en cause le système statutaire, tout en organisant des cadres d'emplois par filières de métiers qui seraient, à terme, communs aux trois versants de la fonction publique. Il fallait donc dialoguer pour moderniser, sans précipitation ni passage en force. Dans le cadre de la LOLF, la Gestion des Ressources humaines ne pouvait s'exercer sur la gestion des agents que par la «performance », ce qui a suscité une forte résistance à la fois de l'administration et des représentants des personnels. La CFDT acceptait de passer d'une culture de moyens à une culture de résultats, à condition que les missions de l'administration soient préalablement définies. D'où son inquiétude à voir la GRH s'appliquer dans un contexte de contraintes budgétaires et de réductions d'effectifs.

\footnotetext{
${ }^{17}$ Fonctions Publiques Informations. CFDT, 31 décembre 2007.

18 «Moderniser la GRH dans la Fonction publique », Revue Française des Finances publiques, nov. 2008, p. 6871.
} 
En fait, le dialogue social se portait toujours mal $^{19}$, car la forme l'a emporté sur le fond, le compromis n'existe pas et le système privilégie le «tout-protestataire », qui laisse les mains libres aux employeurs publics. Le statut des fonctionnaires doit être préservé, mais il repose sur une conception dépassée des relations sociales, et il faut accorder une place à la négociation contractuelle avec des accords majoritaires. La CFDT a demandé en vain une reconnaissance juridique et non plus seulement politique des accords conclus et considère que le paritarisme ne doit pas être numérique, mais doit renvoyer à la «prise de décision conjointe » des représentants des personnels et des employeurs. Enfin, l'UFFA estime que subordonner la politique salariale au non remplacement d'un fonctionnaire partant à la retraite sur deux est un troc inacceptable. De même que la loi relative à la mobilité et aux parcours professionnels multiplie les facteurs de précarisation ${ }^{20}$.

\section{Conclusion}

Ces principales critiques faites par les organisations syndicales correspondent au sentiment d'une grande majorité de fonctionnaires tel qu'il s'exprimait dans un sondage de novembre $2008^{21}$. Ainsi, de $70 \%$ à $90 \%$ d'entre eux estimaient que les réformes en cours ne répondaient pas aux attentes des usagers, pas plus qu'aux attentes des agents de l'Etat. Pour eux, il s'agissait, en fait, d'un affichage politique, sans concertation interne, pour lequel ils se sentaient insuffisamment informés des objectifs et dont ils estimaient que cela n'améliorerait, ni les conditions de travail, ni le service rendu aux usagers. Cependant, une petite majorité (51\%) pensait que la réforme de l'Etat était indispensable et urgente, tout en approuvant à $76 \%$ que les réformes nécessaires soient échelonnées dans le temps.

Philippe Séguin, en juillet 2009, disait tout haut ce que beaucoup de hauts fonctionnaires pensent tout bas : la LOLF avait fait naître de grands espoirs mais, huit ans après, les résultats se font attendre. De même, les effets de la RGPP sont très en deçà des enjeux, car la crise n'explique pas tout. Les déficits sont structurels et il faut, non seulement maîtriser les dépenses, mais ne pas maintenir des baisses d'impôts au-delà de nos moyens ${ }^{22}$.

\footnotetext{
${ }^{19}$ Interview de B. Jumel, Cahiers de la Fonction publique, avril 2009, p. 3-4.

${ }^{20}$ Communiqués de presse des 25 juin et 8 juillet 2009 .

${ }^{21}$ Acteurs publics. Ineum Consulting. Enquête sur le moral des fonctionnaires, portant sur 3.764 fonctionnaires, du 3 au 13 novembre 2008. www.acteurspublics.com

${ }^{22}$ Rencontres de la Modernisation de l'Etat, 8 juillet 2009. www.acteurspublics.com
} 
Les syndicats, devant une «réforme décomplexée » ${ }^{23}$, sont face à un dilemme : comment gérer les conséquences de ces réformes tout en récusant leurs objectifs ? Et comment se prêter à un dialogue sur le dialogue alors que se mettent en place, sans concertation, des changements irréversibles? Car, malgré les efforts méritoires de la Direction générale de l'Administration et de la Fonction publique, et contrairement aux résultats espérés, les effets pervers en matière de charge de travail, de désorganisation et de démotivation, ne se sont pas fait attendre.

Même si la crise du syndicalisme ne date pas des dernières années, face à ces évolutions à marche forcée, les organisations de fonctionnaires sont de plus en plus désemparées. Malgré leur volonté de rénover le dialogue social et de revoir les règles de la représentativité, la majorité d'entre elles, après avoir approuvé les accords de Bercy en 2008, attend le vote de la loi au Parlement. Pour survivre et retrouver quelques marges de manœuvre, elles savent qu'elles devront se réorganiser profondément par des regroupements, au moins électoraux. L'avenir est, pour elles, de plus en plus incertain.

Les deux incertitudes concernant le rôle des syndicats d'une part, et la place de l'administration et de l'Etat dans un monde nouveau, se cumulent. La question qui se pose aujourd'hui à l'observateur est de savoir s'il n'est pas trop tard pour remettre à plat, dans la transparence, les missions et objectifs de l'administration, comme le réclament depuis longtemps les syndicats de fonctionnaires, qui ne sont évidemment pas les seuls concernés. On a voulu réinventer l'Etat, sans doute aurait-il aussi fallu réinventer un intérêt général ${ }^{24}$ et y redéfinir la place des syndicats?

Août 2009.

\footnotetext{
${ }^{23}$ Bezès (Philippe). Réinventer l'Etat. Les réformes de l'administration française (1962-2008).Paris, PUF, 2009, $520 \mathrm{p}$.

${ }^{24}$ Pour une mise en perspective historique :

Rosanvallon (Pierre), La légitimité démocratique. Impartialité, réflexivité, proximité, Paris, Seuil, 2008,368 p.
} 


\section{Résumé}

Panorama des argumentaires de la presse syndicale Force Ouvrière, CGT et CFDT sur les principales réformes managériales depuis 2002: Statut, Dialogue social, Loi organique relative aux lois de finances, Révision générale des politiques publiques. Toutes les organisations se situent plus ou moins entre ces trois «modèles »: d'une résistance sans concession, à la défense d'une fonction publique citoyenne, ou à des analyses centrées sur des méthodes jugées inadéquates.

\section{Mots clés :}

Syndicats, statut, dialogue social, loi organique relative aux lois de finances (LOLF), révision générale des politiques publiques (RGPP). 